\section{POSSIBILITY OF IMMUNOTHERAPY FOR THE GLIOBLASTOMA PATIENTS WITH O6-METHYL-GUANINE DNA METHYLTRANSFERASE (MGMT) EXPRESSION OR PROMOTER UNMETHYLATED}

${ }^{1}$ Yoshihiro Kushihara*, ${ }^{2}$ Yukari Kobayashi, ${ }^{2}$ Koji Nagaoka, ${ }^{2}$ Kazuhiro Kakimi. ${ }^{1}$ The University of Tokyo, Bunkyo-ku, Japan; ${ }^{2}$ The University of Tokyo Hospital, Bunkyo-ku, Japan

Background It has been widely accepted that O6-methyl-guanine DNA methyltransferase (MGMT) promoter methylation in glioblastoma is associated with a benefit from temozolomide (TMZ) treatment. MGMT is a DNA repair protein that removes the cytotoxic O6-methylguanine (O6MG) DNA lesions generated by TMZ; thereby, MGMT expression is mechanistically linked to TMZ resistance. However, thus far, there is no effective treatment for these patients with MGMT promoter unmethylated. Therefore, a new treatment for GBM patients with MGMT expression is urgently needed. ${ }^{1}{ }^{2}$ To this end, we examined the tumor microenvironment in GBM with or without MGMT expression.

Methods Based on The Cancer Genome Atlas (TCGA) primary GBM cohort, the tumor-infiltrating lymphocyte expression level was calculated using the CIBERSORTx algorithms and the single-sample Gene Set Enrichment Analysis (ssGSEA) method. Furthermore, the differential expression gene analysis was conducted and pathway analysis was performed using Ingenuity Pathway Analysis (IPA). The results were validated using the GBM cohort from the Chinese Glioma Genome Atlas (CGGA) database. In addition, tumor-infiltrating lymphocytes (TILs) were isolated from 13 surgically removed primary GBM tumors in our institution. Their responses to autologous tumors were evaluated by IFN $\gamma$ ELISA.

Results T cells CD8 score by CIBERSORTx was significantly higher in the MGMT-high tumor. Similarly, ssGSEA scores for activated CD8 T cell, Macrophage, activated B cell, and Type $1 \mathrm{~T}$ helper cell were significantly higher in the MGMT-high tumor. Conversely, T cells CD4 naive was significantly higher in the MGMT-low tumor. These results indicate that more immune cell infiltration is associated with MGMT-high tumors. Consistently, tumor-reactive TILs were detected in the MGMT-high tumor. Pathway analysis showed that oxidative phosphorylation (OXPHOS) was highly enriched in the MGMT-high tumor.There were many CD8 T cells and tumorreactive $\mathrm{T}$ cells in the MGMT-high tumors. However, it has been reported that anti-PD-1/PD-L1 monotherapy was not effective in glioblastoma. In this study, we demonstrated that OXPHOS was highly activated in the MGMT-high tumors. Thus, metabolic therapy can be combined with immunotherapy in these MGMT-high tumors to enhance anti-tumor immune responses.

Conclusions Although MGMT-high tumors are resistant to $\mathrm{TMZ}$, the existence of immune cell infiltration in the tumor microenvironment of MGMT-high tumors suggest the potential of immunotherapy in these patients.

\section{REFERENCES}

1. Stupp R, Hegi ME, Mason WP, van den Bent MJ, Taphoorn MJB, Janzer RC, Ludwin SK, Allgeier A, Fisher B, Belanger K, Hau P, Brandes AA, Gijtenbeek J, Marosi C, Vecht CJ, Mokhtari K, Wesseling P, Villa S, Eisenhauer E, Gorlia T, Weller $M$, Lacombe D, Cairncross Jy, Mirimanoff R-O, European Organisation for Research and Treatment of Cancer Brain Tumour and Radiation Oncology Groups; National Cancer Institute of Canada Clinical Trials Group. Lancet Oncol 2009;10 (5):459-66

2. Wick W, Weller $M$, van den Bent $M$, Sanson $M$, Weiler $M$, von Deimling $A$, Plass C, Hegi M, Platten M, Reifenberger G. Nat Rev Neurol 2014;;10(7):372-85.
Ethics Approval G3545-(26)

Consent Written informed consent was obtained from the patient for publication of this abstract and any accompanying images. A copy of the written consent is available for review by the Editor of this journal.

http://dx.doi.org/10.1136/jitc-2021-SITC2021.647 